Thiago Santos de Souza ${ }^{1}$

Liliam Silva das Virgens ${ }^{1}$

\section{Saúde do trabalhador na Atenção Básica: interfaces e desafios}

\author{
Workers' health in primary health care: interfaces \\ and challenges
}

${ }^{1}$ Programa de Residência Multiprofissional em Saúde da Família. Escola Bahiana de Medicina e Saúde Pública. Salvador, BA, Brasil.

Contato:

Thiago Santos de Souza

E-mail:

thiago_fst@yahoo.com.br

O trabalho não foi subvencionado.

Os autores declaram não haver conflitos de interesse.

\section{Resumo}

A construção da Saúde do Trabalhador (ST) no Sistema Único de Saúde (SUS) representa uma conquista da classe trabalhadora ao longo de décadas. O processo de desenvolvimento desse campo no contexto brasileiro das relações produção/trabalho, ambiente e saúde é abordado neste artigo no âmbito da Atenção Básica. Objetivo: Refletir acerca das interfaces da ST na Atenção Básica do sistema de saúde brasileiro. Método: Revisão de documentos oficiais e publicações na área, organizados em duas categorias de análise: panorama histórico da ST e da ST na Atenção Básica. Resultados: As categorias de análise possibilitaram estabelecer o resgate histórico da ST no SUS e refletir acerca de elementos da ST na Atenção Básica, como: território e ferramentas de atenção à saúde; competência das equipes da atenção básica; vigilância em saúde do trabalhador; relações de produção e saúde ambiental; práticas educativas em ST; vulnerabilidades do trabalhador da saúde e implementação de experiências institucionais. Conclusão: A incorporação da ST na Atenção Básica caracteriza-se como elemento para redefinição de práticas e mudança no modelo assistencial.

Palavras-chave: saúde do trabalhador; atenção básica à saúde; territorialização; saúde ambiental; vigilância em saúde do trabalhador.

\begin{abstract}
The introduction of worker's health in the Brazilian National Health System (SUS) represents decades of working class conquest. The development process of the worker's health field in the Brazilian production/labor, environment and health relations context is addressed within the primary care setting. Objective: to reflect on the occupational health interfaces of the Brazilian primary health system. Method: review of official documents and publications related to the area, both organized into two categories of analysis: worker's health historical overview and worker's health within primary care. Results: Through these categories of analysis it was possible to produce an historical revival of worker's health in SUS and reflect on elements of worker's health in primary care, such as: territory and health care tools; primary care teams competence, worker's health surveillance; production and environmental health relations; educational practices in worker's health; health worker's vulnerabilities and institutional experiences. Conclusion: worker's health incorporation in primary care is characterized by an element to reset and change practices in the healthcare model.
\end{abstract}

Keywords: worker's health; primary health care; territorial basis organization; environmental health; worker's health surveillance. 


\section{Introdução}

A construção do campo da Saúde do Trabalhador (ST) no Sistema Único de Saúde (SUS) reflete o acúmulo técnico-científico ao longo da história, assim como a correlação das forças sociais, disputadas entre o movimento organizado dos trabalhadores e os setores políticos institucionais das esferas de gestão e das instituições produtoras de conhecimento (DIAS; HOEFEL, 2005).

Em meio ao conjunto de inovações tecnológicas e organizacionais propostas pela reestruturação produtiva na sociedade capitalista, a pauta trabalho e, consequentemente, a saúde para o trabalhador tornam-se elementos indispensáveis para a manutenção de condições dignas nos processos/ambientes de trabalho e na potencialização da vida dos sujeitos (LARA, 2011).

A ST é caracterizada como campo de práticas e conhecimentos que buscam conhecer e intervir nas relações de trabalho e saúde-doença. Ela ainda pode ser entendida enquanto prática teórica interdisciplinar (geração de conhecimento) e prática político-ideológica (superação das relações de poder e conscientização dos trabalhadores), desenvolvidas por diversos atores situados em lugares sociais distintos e informados por uma perspectiva comum (LACAZ, 2007; MINAYO-GOMEZ; THEDIM-COSTA, 1997).

A reorientação do modelo assistencial a partir de 1978, após a Conferência Internacional de Cuidados Primários, tem possibilitado a fertilização das discussões da ST na Atenção Básica (AB). Para tanto, urge a importância da aproximação das equipes de saúde, em especial as equipes de saúde da família, junto às necessidades sociais estabelecidas nos territórios pelos trabalhadores (SANTOS; RIGOTTO, 2011).

A recente publicação da Política Nacional de Saúde do Trabalhador e da Trabalhadora (PNST) reitera a atenção primária em saúde como ordenadora da rede de atenção no SUS, ao passo que objetiva promover a integração com os demais componentes da vigilância em saúde e promover mudanças substanciais nos processos de trabalho (BRASIL, 2012).

Diante desse quadro, faz-se necessário entender o contexto em que a ST vem se conformando e o modo como se estabelecem as práticas voltadas aos trabalhadores dentro da atenção primária à saúde. Tal panorama reforça a importância da produção de experiências na área, colaborando, assim, com o amadurecimento de ações no referido campo.

O presente artigo tem como objetivo refletir sobre as interfaces da ST na atenção básica do sistema de saúde brasileiro.

\section{Método}

Trata-se de um estudo de revisão de literatura do tipo narrativa, com base nas produções bibliográficas realizadas no período de 1986 a 2012. Este recorte de vinte e seis anos foi necessário diante do marco de publicações de referência na área da saúde do trabalhador, como o relatório da VIII Conferência Nacional de Saúde (BRASIL, 1986).

A seleção dos artigos foi realizada através das bases de dados Scientific Electronic Library Online (SciELO) e Google Scholar, a partir dos descritores: saúde do trabalhador, atenção básica à saúde, territorialização, saúde ambiental, vigilância em saúde do trabalhador. A amostra seguiu os seguintes critérios de inclusão: idioma de publicação (todas as produções deveriam estar no idioma português); ano de publicação (as produções deveriam compreender o recorte de tempo estabelecido); modalidades da produção científica (foram incluídas as seguintes modalidades de trabalho: estudos de casos, estudos avaliativos, descritivos e de revisão). A busca bibliográfica também utilizou documentos oficiais, como leis, políticas, portarias, relatórios, normas e manuais técnicos disponibilizados no site do Ministério da Saúde, assim como capítulos de livros pertinentes ao tema. Foram excluídas as produções bibliográficas em outros idiomas, as que não possuíam texto completo e aquelas que não permitiram reflexões que aproximassem os campos da ST com a AB.

Este processo envolveu, primeiramente, a leitura dos resumos para se identificar a pertinência com o objeto estudado e, posteriormente, a seleção dos artigos na íntegra. Após a composição do banco de dados, foi realizado um fichamento para melhor sistematização das informações distribuídas nas dimensões: ano de publicação, objeto do estudo, periódico de indexação, desenho do estudo e principais achados da pesquisa.

A fim de descrever o estado da arte da ST no SUS, particularmente na $\mathrm{AB}$, e discuti-lo sob o ponto de vista teórico, o desenvolvimento do artigo foi organizado em duas categorias de análise: panorama histórico da ST e ST na AB.

A primeira categoria colabora com a introdução da temática, apresentando um recorte da construção do campo ST no SUS com a publicação de leis, propósito das conferências na área, a formação da Rede Nacional de Atenção Integral à Saúde do Trabalhador, bem como a recente publicação da PNST.

A segunda categoria dispõe de subcategorias distribuídas, conforme a identificação e a agregação dos conteúdos comuns entre as referências. São elas: território e ferramentas de atenção à saúde; compe- 
tência das equipes da Atenção Básica; vigilância em saúde do trabalhador; relações de produção e saúde ambiental; práticas educativas em ST; vulnerabilidades do trabalhador da saúde; e implementação de experiências institucionais.

\section{Resultados e discussão}

\section{Panorama histórico da Saúde do Trabalhador}

A ST ganhou evidência durante o movimento da Reforma Sanitária brasileira. O relatório da VIII Conferência Nacional de Saúde, em 1986, já apontava que o trabalho em condições dignas, o conhecimento e o controle dos trabalhadores sobre processos e ambientes de trabalho é um pré-requisito central para o pleno exercício do acesso à saúde (BRASIL, 1986).

A partir de então, houve um crescente avanço legal na área, que teve seu início com a incorporação da ST e da proteção ao ambiente de trabalho pela Constituição Federal (BRASIL, 1988). Em 1990, a Lei no 8.080 reconheceu o trabalho como um dos fatores determinantes e condicionantes da saúde e atribuiu ao SUS a responsabilidade de coordenar as ações de ST no país (BRASIL, 1990).

Desde a década de 1980 até o momento, foram realizadas três Conferências Nacionais de Saúde do Trabalhador. A primeira ocorreu em 1986 e marcou, politicamente, a instituição deste campo (FUNDAÇÃO OSWALDO CRUZ, 1987). A segunda, em 1994, quando foi deliberada a unificação das ações de ST no SUS (BRASIL, 1994). Na última conferência, realizada em 2005, foram estabelecidas tentativas de melhorar as relações interministeriais, porém, em algumas resoluções, observou-se a repetição de proposições anteriores voltadas à lógica da saúde ocupacional (LACAZ, 2007).

Com o propósito de implementar ações assistenciais, de vigilância e de promoção da saúde no SUS, surge, em 2002, a Rede Nacional de Atenção Integral à Saúde do Trabalhador (Renast). Esta possui, como eixo principal para articulação das ações, os Centros de Referência em ST, que preveem a organização da rede sentinela de notificação, a organização dos fluxos de informações e os atendimentos aos trabalhadores em todos os níveis de atenção do SUS. A Renast não visa a criar outro modelo de atenção, mas sim o qualificar, orientar para que o sistema funcione na perspectiva da ST (DIAS; HOEFEL, 2005).

Em 2012, foi instituída a PNST, o que representa um avanço histórico na área, marcada pela luta por direitos da classe trabalhadora. Vale ressaltar que a política está em consonância com a Política Nacional de Segurança e Saúde no Trabalho, instituída por meio do Decreto № 7.602, de 7 de novembro de 2011, e tem como uma de suas estratégias a estruturação da Renast (BRASIL, 2011a, 2012).

A PNST tem como objetivos: fortalecer a vigilância em ST e a integração com os demais componentes da vigilância em saúde; promover saúde, ambientes e processos de trabalhos saudáveis; garantir a integralidade na atenção à ST; ampliar o entendimento e conceber a ST como uma ação transversal; incorporar a categoria trabalho como determinante do processo saúde-doença; assegurar que a identificação da situação do trabalho dos usuários seja considerada nas ações e nos serviços de saúde do SUS; e assegurar a qualidade da atenção aos trabalhadores usuários do SUS (BRASIL, 2012).

Mesmo com esses avanços, um dos desafios da ST é conseguir com que o conjunto de trabalhadores e gestores do SUS incorpore, na sua prática cotidiana, a compreensão do trabalho enquanto um dos determinantes do processo saúde-doença e da necessidade do envolvimento de todo o sistema de saúde para garantir o cuidado integral aos trabalhadores (DIAS et al., 2011). Ademais, faz-se necessário a constituição de práticas de atenção à saúde que transcendam a abordagem individual curativa e apontem para abordagens interdisciplinares e intersetoriais (LOURENÇO; BERTANI, 2007)

\section{Saúde do trabalhador na atenção básica}

Definida como prioridade do pacto pela vida, a $\mathrm{AB}$ é considerada como porta de entrada preferencial do sistema de saúde, sendo entendida por:

\footnotetext{
Um conjunto de ações de saúde, no âmbito individual e coletivo, que abrange a promoção e a proteção da saúde, a prevenção de agravos, o diagnóstico, o tratamento, a reabilitação, redução de danos e a manutenção da saúde, com o objetivo de desenvolver uma atenção integral que impacte na situação de saúde e autonomia das pessoas e nos determinantes e condicionantes de saúde das coletividades. (BRASIL, 2011b, Anexo I)
}

De acordo com a história, a implantação de serviços de ST no SUS pode ser entendida em momentos distintos, considerando-se o engajamento dos atores sociais envolvidos (MINAYO-GOMEZ; LACAZ, 2005).

As experiências de ST na AB têm início nos anos 1980, no estado de São Paulo. A pressão de setores do movimento sindical pela exigência de uma atenção dos serviços públicos para os problemas de saúde dos trabalhadores culminaram com a criação dos Programas de Saúde do Trabalhador na rede básica. Dentre seus pressupostos, estavam a promoção de uma gestão participativa, acesso a informações de saúde, desenvolvimento de ações de vigilância e visualização 
do trabalhador a partir de sua inserção no processo produtivo (LACAZ, 2007).

Contudo, as dificuldades culturais, ideológicas e políticas colaboraram com amarras na expansão do campo e no estabelecimento de ações eficazes voltadas à ST no SUS e na $\mathrm{AB}$ ao longo das três últimas décadas. Dessa forma, são apresentadas abaixo subcategorias relacionadas à ST na $\mathrm{AB}$ visando a colaborar com subsídios para transformação das práticas.

\section{Território e ferramentas de atenção à saúde}

O território é caracterizado por uma população específica, vivendo em tempo e espaço determinados, com problemas de saúde definidos, mas quase sempre com condicionantes e determinantes que emergem de um plano mais geral. Esse espaço apresenta, além de uma delimitação espacial, um perfil histórico, demográfico, epidemiológico, administrativo, tecnológico, político, social e cultural que o caracteriza como um território em permanente construção (GONDIM et al., 2008).

A aproximação dos trabalhadores da saúde com o território pode ocorrer através da territorialização. Este é um importante instrumento de organização dos processos de trabalho e práticas de saúde. Entretanto, muitas vezes, sua utilização na $\mathrm{AB}$ reduz-se aos diagnósticos das condições de vida e situação de saúde da população, não sendo abordados diversos outros elementos por parte dos profissionais da saúde (SANTOS; RIGOTTO, 2011).

A Estratégia Saúde da Família é a principal ferramenta de atenção à saúde voltada para o reordenamento do modelo assistencial. Possui como objetivo prestar o acompanhamento às famílias de uma área geográfica delimitada e com população adscrita. Baseia-se no trabalho compartilhado entre equipes multiprofissionais, compreendendo os determinantes sociais da saúde e o perfil epidemiológico da população e atuando sobre eles. Orienta-se pelos princípios da universalidade, equidade, acessibilidade, vínculo, continuidade do cuidado, responsabilização, integralidade da atenção, humanização e participação social (BRASIL, 2011b).

Outra ferramenta de atenção à saúde, lançada em 2008 pelo Ministério da Saúde, foi intitulada Núcleos de Apoio à Saúde da Família (NASF). Esse arranjo organizacional tem com objetivo potencializar e ampliar a abrangência e a diversidade das ações da $\mathrm{AB}$, bem como sua resolutividade, apoiando a inserção da Estratégia Saúde da Família na rede. O NASF possui um grupo interdisciplinar que atua em parceria com os profissionais da equipe de referência, compartilhando práticas em saúde, nos territórios de responsabilidade das unidades, sendo operacionalizado pelo apoio matricial (BRASIL, 2008).
As práticas de ST no NASF ainda apresentam-se incipientes mediante as diretrizes propostas pelo Ministério da Saúde, em que apenas consta a necessidade de firmar parcerias com os centros de referência em ST (BRASIL, 2010). Mesmo diante dessa limitação, é importante destacar a diversidade de saberes profissionais que compõem os núcleos e seu grande potencial para elaborar propostas voltadas aos trabalhadores das áreas de abrangência das unidades.

\section{Competências das equipes da Atenção Básica}

A realização de ações em ST na AB deve partir da identificação, no território, da população economicamente ativa (por sexo e faixa etária), do mapeamento das atividades produtivas existentes na área, da identificação dos integrantes das famílias que sejam trabalhadores, da observação dos vínculos empregatícios, dos desempregados e dos que iniciam o trabalho precocemente. Deve ainda levantar os riscos para a saúde dos trabalhadores, da população e do meio ambiente, além de acidentes e/ou doenças relacionados ao trabalho (BRASIL, 2001).

Para tanto, compete aos serviços da rede básica: organizar e analisar os dados obtidos em visitas domiciliares; desenvolver programas de educação em ST; incluir o item ocupação e ramo de atividade em toda ficha de atendimento individual de crianças acima de 5 anos, adolescentes e adultos; planejar e executar ações de vigilância nos locais de trabalho; desenvolver, juntamente com a comunidade e instituições públicas, ações para a solução dos problemas encontrados; e considerar o trabalho infantil como alerta epidemiológico (BRASIL, 2001).

Em relação aos casos de acidente ou doença relacionados ao trabalho, a equipe de saúde deve: realizar a condução clínica dos casos de menores complicações; efetuar encaminhamento dos casos de maior complexidade para serviços especializados em ST; realizar a notificação nos sistemas de informação; investigar o local de trabalho; prestar orientações trabalhistas e previdenciárias; solicitar à empresa a emissão da Comunicação de Acidente de Trabalho; e discutir com o trabalhador as causas de seu adoecimento (BRASIL, 2001).

Apesar de muitos profissionais reconhecerem a importância do trabalho para a saúde e da existência de um caderno temático com informações voltadas à ST na $\mathrm{AB}$, sabe-se que, na prática dos serviços, muitas ações em ST são efetuadas pontualmente ou mesmo inexistem no planejamento das equipes.

Dessa forma, é de suma importância que os trabalhadores da saúde estejam atentos e capacitados para realizarem: o reconhecimento dos processos produtivos no território; o mapeamento das principais ativida- 
des exercidas pelos sujeitos na área adscrita; o levantamento dos riscos ocupacionais; a identificação do trabalho formal/informal e a compreensão dos problemas ambientais gerados pelos atos laborativos e a intervenção sobre eles (SANTOS; RIGOTTO, 2011).

Pode-se dizer que a inserção efetiva das ações de ST no SUS está diretamente relacionada à possibilidade de sua assimilação pela AB. Além dos fatores organizacionais, o crescimento do trabalho informal, familiar e em domicílio reforça o papel da $A B$ de fazer chegar ações de saúde o mais próximo possível de onde as pessoas vivem e trabalham. O acolhimento dos trabalhadores na porta de entrada do sistema, a investigação do trabalho como fator determinante dos processos saúde/doença e a avaliação de situações de risco no trabalho são possibilidades concretas no nível primário de atenção (DIAS; HOEFEL, 2005).

\section{Vigilância em Saúde do Trabalhador}

Em resposta à demanda crescente por uma nova concepção de saúde que pudesse responder à complexidade emergente dos problemas da população, ocorreu, em 1986, na cidade de Ottawa, a I Conferência Internacional sobre Promoção da Saúde. A partir desta, propostas de atuação no setor foram desenvolvidos, dentre elas a promoção da saúde (ORGANIZAÇÃO PANAMERICANA DA SAÚDE, 1986).

Segundo a carta de Ottawa, a promoção da saúde está associada a um conjunto de valores, como: qualidade de vida, saúde, cuidado, equidade, preservação dos recursos naturais, cidadania e participação social. Refere-se também a uma combinação de estratégias de responsabilização múltipla da sociedade, incluindo: políticas públicas saudáveis, reforço da ação comunitária, desenvolvimento de habilidades pessoais, reorientação do sistema de saúde e parcerias intersetoriais (ORGANIZAÇÃO PANAMERICANA DA SAÚDE, 1986).

Sob a influência desse cenário, a ST também precisou gerar novas respostas sociais aos problemas elencados pelos trabalhadores, propondo-se a ir além daqueles restritos à relação saúde-doença e às intervenções insuficientes no controle das condições e dos processos de trabalho (ALVES, 2003).

Com base nessas concepções, surge a vigilância em saúde do trabalhador (Visat) no SUS, definida pelo Ministério da Saúde como:

Uma atuação contínua e sistemática, ao longo do tempo, no sentido de detectar, conhecer, pesquisar e analisar os fatores determinantes e condicionantes dos agravos à saúde, relacionados aos processos e ambientes de trabalho, em seus aspectos tecnológico, social, organizacional e epidemiológico, com a finalidade de planejar, executar e avaliar intervenções sobre esses aspectos, de forma a eliminá-los e controlá-los. (BRASIL, 1998, Anexo I)
Um importante marco legal, relacionado à Visat, foi a publicação da Portaria no 3.252, de 22 de dezembro de 2009 (BRASIL, 2009), pois favoreceu a ampliação do debate em torno das ações de vigilância em ST no âmbito da $\mathrm{AB}$, de forma articulada aos outros componentes da vigilância em saúde. Nesse documento, a Visat busca a promoção da saúde e a redução da morbimortalidade da população trabalhadora por meio da integração de ações que intervenham nos agravos e em seus determinantes decorrentes dos modelos de desenvolvimento e processo produtivos.

Diferente da vigilância em saúde, seu objeto de investigação e intervenção é fruto da relação entre o processo de trabalho e saúde. Suas ações buscam a articulação sanitária no sentido da promoção, da proteção e da atenção à saúde dos trabalhadores, direcionadas aos fatores de risco e às condições de trabalho, vigilância sobre danos ou efeitos e seus determinantes tecnológicos e sociais (MACHADO, 1997; NOBRE et al., 2011).

Para que tais modalidades da vigilância operem, são necessários: produção de informações, conhecimentos, tecnologias de intervenção, desenvolvimento de abordagens pedagógicas e formativas, estratégias de difusão, comunicação e o fortalecimento do controle social (NOBRE et al., 2011).

A vigilância em saúde do trabalhador rompe com a abordagem hegemônica que vincula a doença a um agente específico ou a um grupo de agentes existentes no ambiente de trabalho. Pauta-se na concepção de que a saúde para o trabalhador não significa apenas a ausência de doenças ocupacionais e acidentes de trabalho, mas também, e principalmente, a transformação dos processos de trabalho em seus diversos aspectos, que visem à inserção do trabalhador no processo produtivo, que seja potencializadora de saúde e vida (ALVES, 2003).

Para que a vigilância em saúde do trabalhador se configure como elemento estruturante do SUS, faz-se necessário que suas ações, incluindo a promoção da saúde, estejam inseridas no cotidiano das equipes da $\mathrm{AB}$ como condição obrigatória para a construção da integralidade na atenção (BRASIL, 2009).

\section{Relações de produção e saúde ambiental}

As inter-relações produção/trabalho, ambiente e saúde são determinadas pelo modo de produção de cada sociedade, sendo a principal referência para se entender as condições de vida, o perfil de adoecimento e morte, a vulnerabilidade de certos grupos sociais e a degradação ambiental (DIAS et al., 2009).

Para a produção de bens e riquezas, são requeridas matérias-primas, trabalho e tecnologia. Nessa interface, a economia se sustenta pela exploração 
da natureza e do trabalhador, gerando a degradação ambiental e as cargas biológicas, sociais, psíquicas, econômicas, políticas, culturais que afetam a saúde dos trabalhadores e de suas famílias e produzem desigualdades sociais e ambientais (DIAS et al., 2009).

As críticas ao modelo de desenvolvimento puramente econômico e seus impactos sobre as condições de vida, saúde, doença e ambiente têm aproximado os campos da ST e da saúde ambiental (FREITAS; PORTO, 2006).

A inserção da ST e da saúde ambiental na $A B$ encontou obstáculos na diversidade das situações e dos arranjos institucionais e na pouca tradição em ações de vigilância nesse nível de atenção, quando comparadas às práticas assistenciais curativas (DIAS et al., 2009).

Para que ocorra o andamento dessas práticas, não basta acrescentar mais uma atribuição às já sobrecarregadas equipes de saúde da família e NASF. É importante que as tarefas sejam redimensionadas, as equipes, capacitadas, valorizadas, que disponham de suporte de recursos físicos, materiais e organizacionais pelas secretarias de saúde, além do bom funcionamento da rede e dos fluxos de referência e contrarreferência.

\section{Práticas educativas em ST}

Investimentos na formação profissional e na educação permanente em ST ganharam relevância estratégica com o avanço da descentralização da saúde, especialmente pelos desafios de implantação/implementação da Renast (AYRES; SANTOS, 2011).

Entre os anos de 2007 e 2008, a Diretoria de Vigilância e Atenção à Saúde do Trabalhador/Centro de Referência Estadual em Saúde do Trabalhador da Bahia (Divast/Cesat) apresentou uma proposta de educação permanente direcionada ao fortalecimento da Renast no estado (AYRES; SANTOS, 2011).

Neste processo, ocorreram momentos presenciais e oficinas de trabalho descentralizadas voltadas aos trabalhadores do Cesat, dos centros de referência em ST e das diretorias regionais de saúde. Foram abordados elementos nas áreas de atenção, gestão e vigilância, valorizando a vivência de cada sujeito, do coletivo, das instituições e dos serviços no que tange à identificação dos problemas em ST e ao início do enfrentamento de muitos entraves e desafios postos em cena (AYRES; SANTOS, 2011).

Neves et al. (2006) desenvolveram um ensaio colocando em questão o uso da biossegurança enquanto ação educativa. As autoras apontam que a biossegurança é capaz de oferecer contribuições significativas à ST. Todavia, sua ação deve ir além do mero treinamento e transmissão de informação. Sob uma perspectiva de prática transformadora, é necessário que os sujeitos sejam agentes ativos da aprendizagem e tenham suas diferenças e singularidades levadas em conta.

Outro estudo destaca as mudanças ocorridas no estilo de vida de trabalhadores em relação à prevenção dos fatores de risco da hipertensão arterial sistêmica, a partir da aplicação de uma tecnologia educativa em saúde. Essa ferramenta, oriunda das tecnologias leves e proposta por Merhy, contribuiu para a obtenção de maior autonomia e fortalecimento do caráter de agente multiplicador em 16 dos 20 trabalhadores participantes das cinco oficinas educativas (SANTOS; LIMA, 2008).

Apesar de o desenvolvimento e a capacitação de recursos humanos serem uma das estratégias da PNST, poucas evidências relacionadas a práticas educativas específicas à ST na $\mathrm{AB}$ são encontradas.

Conforme a PNST, os profissionais de níveis superior e médio das equipes de vigilância em saúde, saúde da família e dos gestores devem ter a inserção de conteúdos de ST nos diversos processos formativos e estratégias de educação permanente. Além disso, a capacitação para aplicação de protocolos, linhas guias, linhas de cuidado em ST e orientações quanto aos direitos dos trabalhadores devem ser ofertadas (BRASIL, 2012).

\section{Vulnerabilidades do trabalhador da saúde}

Apesar de possuir papel chave dentro da sociedade, os próprios trabalhadores da saúde constituem um grupo vulnerável. O perfil da população atendida nos estabelecimentos origina a sobreposição dos riscos profissionais clássicos (químicos e físicos), dos riscos biológicos emergentes e dos relacionados ao excesso de demanda e polivalência (riscos psicossociais) (ASSUNÇÃO, 2011).

Somando-se a isso, a expansão do emprego na saúde muitas vezes ocorre na ausência de um contrato de trabalho padrão. São crescentes os vínculos não regulamentados, por tempo parcial, tempo determinado, terceirização, subcontratação e até contratos verbais entre prefeituras e profissionais da saúde. Tal contexto reflete em adoecimento, vida com menos segurança nos ambientes ocupacionais, falta de prazer e desmotivação na realização de ações nas diversas ordens, incluindo a ST (ASSUNÇÃO, 2011).

Bezerra e Neves (2010) efetuaram uma revisão de literatura com o objetivo de traçar o perfil da produção científica em ST, de 2001 a 2008. Nesse estudo, verificou-se que a população mais estudada foi a dos profissionais da área de saúde. Entretanto, outra revisão sob autoria de Chiode e Marziale (2006), com intuito de buscar evidências sobre os 
riscos ocupacionais a que estão submetidos os trabalhadores que atuam na saúde pública, evidenciou que apenas $4,3 \%$ das publicações enfocaram riscos ocupacionais voltados ao contexto das unidades da rede básica.

Apesar de ainda não ser encontrada na literatura uma revisão atual sobre a produção voltada aos riscos e às vulnerabilidades do trabalhador da $\mathrm{AB}$, sabe-se que o número de artigos cresce vertiginosamente.

Como exemplo, são citados os estudos de Lancman et al. (2009) acerca das repercussões da violência na saúde mental de trabalhadores do programa Saúde da Família; Braga et al. (2010), referente às condições de trabalho e transtornos mentais comuns em trabalhadores da rede básica de saúde de Botucatu/SP; Trindade et al. (2010), relacionado ao estresse e à síndrome de burnout entre trabalhadores das equipes de saúde da família; Ursine et al. (2010), sobre as condições de trabalho e a qualidade de vida dos agentes comunitários de saúde; e a pesquisa de Fernandes et al. (2012), que versa sobre a relação dos aspectos profissionais na qualidade de vida dos enfermeiros da saúde da família.

\section{Implementação de experiências institucionais}

Mesmo diante de um cenário de obstáculos, iniciativas práticas vêm sendo desenvolvidas na aproximação da ST com a AB. No estado de São Paulo, a Fundação Seade realizou, nos anos de 1995, 1997 e 1999, um estudo em 520 municípios com objetivo de caracterizar a implantação da descentralização das ações de ST. Foi identificado que, em 1999, 26\% das prefeituras paulistas realizavam ações na área, mais frequentemente em municípios maiores e em gestão plena do sistema. As práticas mais comuns foram a vistoria e a fiscalização de ambientes de trabalho e a oferta de serviços de reabilitação física aos acidentados do trabalho (SILVA et al., 2003).

No município de Amparo/SP, a implantação das ações de ST na rede de AB, entre 2003 e 2008, foi marcada por uma proposta de gestão colegiada. O programa de implantação englobou quatro conjuntos de ações: assistência ao trabalhador; Visat; educação em saúde; e capacitação dos profissionais da rede municipal. Segundo as autoras, os aspectos que colaboraram para o sucesso da proposta foram as estratégias de sensibilização adotadas, de educação permanente e de corresponsabilização entre as equipes (DIAS et al., 2011).

Nesse mesmo município, outra experiência foi identificada, agora com objetivo de compreender o apoio matricial em $S T$ na $A B$, realizada no ano de 2008. Em Amparo, as equipes de referência são as próprias equipes de saúde da família, e os apoiadores são as duplas de profissionais do centro de refe- rência em saúde do trabalhador responsáveis, cada qual, por três ou quatro unidades de saúde. Através dessa parceria, o estudo conclui que o apoio matricial pôde contribuir com a mudança no olhar dos profissionais de saúde da família frente aos trabalhadores atendidos e promover a reorganização do trabalho em saúde (SANTOS; LACAZ, 2012).

Em Campinas/SP, foi desenvolvido um estudo com objetivo de avaliar o processo de implantação da diretriz da descentralização da Visat para as 49 unidades básicas do município. Os acidentes e as doenças relacionadas ao trabalho foram utilizados como critérios de avaliação. Observou-se que a descentralização ocorreu de forma parcial, pois as questões clínicas não se transformaram em ações de vigilância. Em muitos casos, ocorreu a subnotificação das doenças relacionadas ao trabalho, e o trabalhador informal não recebeu ação específica de vigilância para seu problema (BALISTA et al., 2011).

As pesquisas referidas acima apresentam grande importância para a área, visto que se mostram como possibilidades viáveis de desvendar a complexidade das ações voltadas à ST e serviram de estímulo a novas produções.

\section{Considerações finais}

Esta pesquisa buscou realizar um resgate histórico da ST no SUS e apresentar elementos do campo da ST na AB, colaborando, assim, para sistematização da produção bibliográfica na área.

Apesar dos avanços conquistados, muitos desafios são postos ao campo, como: o combate ao reducionismo das políticas públicas, aos entraves políticos no âmbito municipal, e ao desconhecimento de muitos gestores frente à concepção e às ações da ST; a consolidação da Renast como política articuladora da rede e vinculada a outras políticas da seguridade social; a necessidade de tratar com responsabilidade o trabalho infantil, o informal, o trabalho de rua e o desemprego; a expansão da atuação para o setor de serviços e agricultura.

Faz-se necessário, ainda, a ampliação dos objetos de estudos e a reformulação de referenciais conceituais e metodológicos; uma formação acadêmica que reconheça a tensa relação entre capital e trabalho; a capacitação dos profissionais da saúde para lidar com os riscos e os agravos relacionados ao trabalho; uma produção científica socialmente referenciada; e movimentos sociais e sindicais atuantes.

A ST no SUS busca criar mecanismos de fortalecimento e construção de novas práticas, em especial na $\mathrm{AB}$, ordenadora do atual modelo de produção do cuidado. Com o enfoque na educação permanente, 
na promoção e na vigilância da saúde, espera-se que os profissionais de saúde da família e dos NASF possam ampliar seu olhar e o escopo de suas ações no território na medida em que desfrutem de condições e recebam qualificação para atuar.

Com a publicação da PNST, cresce a expectativa pela reorganização do sistema de saúde, voltado ao trabalhador, especialmente pela AB. É emergen- te a busca pela formação de redes de atenção que vislumbrem o trabalhador com outro olhar, ao promover acolhimento de suas demandas, dando-lhes significado e uma resposta positiva.

Os desafios são diversos e complexos, porém não se pode esquecer de que muitos dos entraves, no campo da ST, são oriundos da conformação do mundo do trabalho no atual modelo de sociedade vigente.

\section{Contribuições de autoria}

Souza, T. S.: trabalhou na concepção teórica, na elaboração e na redação final do manuscrito. Virgens, L. S.: orientou a pesquisa e contribuiu na revisão final do estudo.

\section{Referências}

ALVES, R. B. Vigilância em saúde do trabalhador e promoção da saúde: aproximações possíveis e desafios. Cadernos de Saúde Pública, Rio de Janeiro, v. 19, n. 1, p. 319-322, 2003.

ASSUNÇÃO, A. A. Condições de trabalho e saúde dos trabalhadores da saúde In: GOMEZ, C. M.; MACHADO, J. M. H.; PENA, P. G. L. (Org.). Saúde do trabalhador na sociedade brasileira contemporânea. Rio de Janeiro: Fiocruz, 2011. p. 453-473.

AYRES, I. B. S. J.; SANTOS, S. L. B. Educação permanente e formação profissional em saúde do trabalhador: desafios e potencialidades. In: NOBRE, L. C. C. (Org.). A saúde do trabalhador na Bahia: história, conquistas e desafios. Salvador: EdUFBA, 2011. p. 249-270.

BALISTA, S. R. R. et al. A atenção à saúde do trabalhador nas unidades básicas de saúde do SUS: estudo de caso em Campinas, São Paulo. Revista Brasileira de Saúde Ocupacional, São Paulo, v. 36, n. 124, p. 216-226, 2011.

BEZERRA, M. L. S.; NEVES, E. B. Perfil da produção científica em saúde do trabalhador. Saúde e Sociedade, São Paulo, v. 19, n. 2, p. 384-394, jun. 2010.

BRAGA, L. C. et al. Condições de trabalho e transtornos mentais comuns em trabalhadores da rede básica de saúde de Botucatu (SP). Ciência \& Saúde Coletiva, Rio de Janeiro, v. 15, supl. 1, p. 1585-1596, jun. 2010.

BRASIL. Conferência Nacional de Saúde, 8. Relatório final. [S.I.: s.n.], 1986. Disponível em: <http:// conselho.saude.gov.br/biblioteca/Relatorios/relatorio_8. pdf $>$. Acesso em: 30 out. 2013.

. Constituição (1988). Promulgada em 05 de outubro de 1988. Brasília, DF, Senado, 1988. Disponível em: <http://www.planalto.gov.br/ccivil_03/ constituicao/constituicaocompilado.htm $>$. Acesso em: 26 set. 2013.
. Decreto $\mathrm{n}^{\circ}$ 7.602, de 7 de novembro de 2011. Dispõe sobre a Política Nacional de Segurança e Saúde no Trabalho (PNSST). Diário Oficial [da] República Federativa do Brasil, Brasília, DF, 08 nov. 2011a. Disponível em: <http://www.planalto.gov.br/ ccivil_03/_Ato2011-2014/2011/Decreto/D7602.htm>. Acesso em: 31 out. 2013.

. Lei $\mathrm{n}^{\mathrm{o}} 8.080$ de 19 de setembro de 1990. Dispõe sobre as condições para a promoção, proteção e recuperação da saúde, a organização e o funcionamento dos serviços correspondentes e dá outras providências. Diário Oficial [da] República Federativa do Brasil, Brasília, DF, 20 set. 1990. Disponível em: < http://www.planalto.gov.br/ccivil_03/ leis/l8080.htm > . Acesso em: 31 out. 2013.

. Ministério da Saúde. Conselho Nacional de Saúde. Conferência Nacional de Saúde do Trabalhador, 2. Relatório Final. [S.I.]: Ministério da Saúde, 1994. Disponível em: < http://bvsms.saude.gov. br/bvs/publicacoes/0207cnst_relat_final.pdf $>$. Acesso em: 20 set. 2013.

. Diretrizes do Núcleo de Apoio à Saúde da Família. Série A. Normas e Manuais Técnicos, Brasília, DF: Ministério da Saúde, 2010. (Cadernos de Atenção Básica, n. 27).

. Portaria MS/GM nº 3.120, de 01 de julho de 1998. Aprova a instrução normativa de vigilância em saúde do trabalhador no SUS. Diário Oficial [da] República Federativa do Brasil, Brasília, DF, 02 jul. 1998. Disponível em: <http://www.cerest.piracicaba. sp.gov.br/site/images/3120___98.pdf>. Acesso em: 31 out. 2013.

. Portaria $\mathrm{n}^{\mathrm{o}}$ 1.679, de 19 de setembro de 2002. Dispõe sobre a estruturação da Rede Nacional de Atenção Integral à Saúde do Trabalhador no SUS. Diário Oficial [da] República Federativa do Brasil, Brasília, DF, 20 set. 2002. Disponível em: < http:// 
dtr2001.saude.gov.br/sas/PORTARIAS/Port2002/Gm/ GM-1679.htm>. Acesso em: 31 out. 2013.

. Portaria GM nº 154, de 24 de janeiro de 2008. Cria os Núcleos de Apoio à Saúde da Família - NASF. Diário Oficial [da] República Federativa do Brasil, Brasília, DF, 2008. Disponível em: < http://portal.saude. gov.br/portal/arquivos/pdf/Portaria_N_154_GMMS. pdf $>$. Acesso em: 31 out. 2013.

. Portaria $\mathrm{n}^{\circ} 3.252$, de 22 de dezembro de 2009. Aprova as diretrizes para execução e financiamento das ações de Vigilância em Saúde pela União, Estados, Distrito Federal e Municípios e dá outras providências. Diário Oficial [da] República Federativa do Brasil, Brasília, DF: Ministério da Saúde, 2009. Disponível em: <http://portal.saude.gov.br/portal/arquivos/pdf/ portaria3252_da_vigilancia_em_saude_0501_atual. pdf $>$. Acesso em: 31 out. 2013.

. Portaria n⿳0 2.488, de 21 de outubro de 2011. Aprova a Política Nacional de Atenção Básica, estabelecendo a revisão de diretrizes e normas para a organização da Atenção Básica, para a Estratégia de Saúde da Família (ESF) e o Programa de Agentes Comunitários de Saúde (PACS). Diário Oficial [da] República Federativa do Brasil, Brasília, DF: Ministério da Saúde, 2011b. Disponível em: <http://www. brasilsus.com.br/legislacoes/gm/110154-2488.html>. Acesso em: 30 out. 2013.

. Portaria no 1.823 , de 23 de agosto de 2012 . Institui a Política Nacional de Saúde do Trabalhador e da Trabalhadora. Diário Oficial [da] República Federativa do Brasil, Brasília, DF: Ministério da Saúde, 2012. Disponível em: <http://bvsms.saude.gov.br/ bvs/saudelegis/gm/2012/prt1823_23_08_2012.html>. Acesso em: 31 out. 2013.

. Saúde do trabalhador. Brasília, DF: Ministério da Saúde, 2001. (Cadernos de Atenção Básica, nº 05).

CHIODI, M. B.; MARZIALE, M. H. P. Riscos ocupacionais para trabalhadores de unidades básicas de saúde: revisão bibliográfica. Acta Paulista de Enfermagem, São Paulo, v. 19, n. 2, p. 212-217, 2006.

DIAS, E. C.; HOEFEL, M. G. O desafio de implementar as ações de saúde do trabalhador no SUS: a estratégia da Renast. Ciência \& Saúde Coletiva, Rio de Janeiro, v. 10, n. 4, p. 817-827, 2005.

DIAS, E. C. et al. Saúde ambiental e saúde do trabalhador na atenção primária à saúde, no SUS: oportunidades e desafios. Ciência \& Saúde Coletiva, v. 14, n. 6, p. 2061-2070, 2009.

DIAS, M. D. A. et al. Saúde do trabalhador na atenção básica: análise a partir de uma experiência municipal. Trabalho, Educação e Saúde, Rio de Janeiro, v. 9, n. 1, p. 137-148, mar./jun. 2011.

FERNANDES, J. S. et al. A relação dos aspectos profissionais na qualidade de vida dos enfermeiros das equipes Saúde da Família. Revista da Escola de
Enfermagem da USP, São Paulo, v. 46, n. 2, p. 404-412, abr. 2012.

FREITAS, C. M.; PORTO, M. F. S. Saúde, ambiente e sustentabilidade. Rio de Janeiro: Fiocruz, 2006.

FUNDAÇÃO OSWALDO CRUZ. Relatório Final da $1^{\text {a }}$ Conferência Nacional de Saúde dos Trabalhadores. Brasília: Ministério da Saúde; Fiocruz - ENSP- Cesteh, 1987.

GONDIM, G. M. M. et al. O território da saúde: a organização do sistema de saúde e a territorialização. In: MIRANDA, A. C. et al. (Org.). Território, ambiente e saúde. Rio de Janeiro: Fiocruz, 2008. p. 237-255.

LACAZ, F. A. C. O campo Saúde do Trabalhador: resgatando conhecimentos e práticas sobre as relações trabalho-saúde. Cadernos de Saúde Pública, Rio de Janeiro, v. 23, n. 4, p. 757-766, abr. 2007.

LANCMAN, S. et al. Repercussões da violência na saúde mental de trabalhadores do Programa Saúde da Família. Revista de Saúde Pública, São Paulo, v. 43, n. 4, p. 682-688, ago. 2009.

LARA, R. Saúde do trabalhador: considerações a partir da crítica da economia política. Revista Katálysis, Florianópolis, v. 14, n. 1, p. 78-85, jan./jun. 2011.

LOURENÇO, E. A. S.; BERTANI, I. F. Saúde do trabalhador no SUS: desafios e perspectivas frente à precarização do trabalho. Revista Brasileira de Saúde Ocupacional, São Paulo, v. 32, n. 115, p. 121-134, 2007.

MACHADO, J. M. H. Processo de vigilância em saúde do trabalhador. Cadernos de Saúde Pública, Rio de Janeiro, v. 13, n. 2, p. S33-S45, 1997.

MINAYO-GOMEZ, C.; LACAZ, F. A. C. Saúde do trabalhador: novas-velhas questões. Ciência \& Saúde Coletiva, Rio de Janeiro, v. 10, n. 4, p. 797-807, 2005.

MINAYO-GOMEZ, C.; THEDIM-COSTA, S. M. F. A construção do campo da saúde do trabalhador: percurso e dilemas. Cadernos de Saúde Pública, Rio de Janeiro, v. 13, n. 2, p. S21-S32, 1997.

NEVES, T. P. et al. Biossegurança como ação educativa: contribuições à saúde do trabalhador. Cogitare Enfermagem, Curitiba, v. 11, n. 1, p. 50-54, jan./abr. 2006.

NOBRE, L. C. C. et al. Vigilância em saúde do trabalhador no sistema único de saúde: práticas e desafios. In: NOBRE, L. C. C. (Org.). A saúde do trabalhador na Bahia: história, conquistas e desafios. Salvador: EdUFBA, 2011.

p. 277-311.

\section{ORGANIZAÇÃO PANAMERICANA DA SAÚDE.}

Primeira Conferência Internacional sobre Promoção da Saúde. Ottawa, novembro de 1986. Disponível em: <http://www.opas.org.br/promocao/uploadArq/Ottawa. pdf $>$. Acesso em: 15 fev. 2012.

SANTOS, A. P. L.; LACAZ, F. A. C. Apoio matricial em saúde do trabalhador: tecendo redes na atenção básica 
do SUS, o caso de Amparo/ SP. Ciência \& Saúde Coletiva, Rio de Janeiro, v. 17, n. 5, p. 1143-1150, maio 2012.

SANTOS, Z. M. S. A.; LIMA, H. P. Tecnologia educativa em saúde na prevenção da hipertensão arterial em trabalhadores: análise das mudanças no estilo de vida. Texto e Contexto Enfermagem, Florianópolis, v. 17, n. 1, p. 90-97, jan./mar. 2008.

SANTOS, A. L.; RIGOTTO, R. M. Território e territorialização: incorporando as relações produção, trabalho, ambiente e saúde na atenção básica à saúde. Trabalho, Educação e Saúde, Rio de Janeiro, v. 8, n. 3, p. 387-406, nov. 2010/fev. 2011.
SILVA, Z. P. et al. Saúde do trabalhador no âmbito municipal. São Paulo Perspectiva, São Paulo, v. 17, n. 1, p. 47-57, mar. 2003.

TRINDADE, L. L. et al. Estresse e síndrome de burnout entre trabalhadores da equipe de Saúde da Família. Acta Paulista de Enfermagem, São Paulo, v. 23, n. 5, p. 684-689, out. 2010.

URSINE, B. L. et al. O Agente Comunitário de Saúde na Estratégia de Saúde da Família: uma investigação das condições de trabalho e da qualidade de vida. Revista Brasileira de Saúde Ocupacional, São Paulo, v. 35, n. 122, p. 327-339, dez. 2010. 